AperTO - Archivio Istituzionale Open Access dell'Università di Torino

\title{
Ecoepidemics overcoming the species-barrier and being subject to harvesting
}

\section{This is the author's manuscript}

Original Citation:

\section{Availability:}

This version is available http://hdl.handle.net/2318/145452

since

Published version:

DOI:10.1093/imammb/dqr026

Terms of use:

Open Access

Anyone can freely access the full text of works made available as "Open Access". Works made available under a Creative Commons license can be used according to the terms and conditions of said license. Use of all other works requires consent of the right holder (author or publisher) if not exempted from copyright protection by the applicable law. 


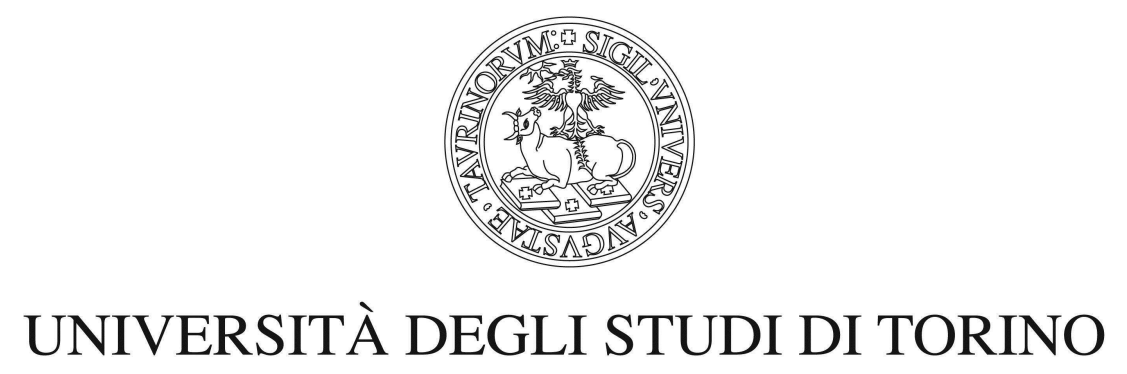

This is an author version of the contribution published on:

S. Chaudhuri, A. Costamagna, E. Venturino. Ecoepidemics overcoming the species-barrier and being subject to harvesting. J. Biol. Phys,. (2011) 37:

91-106, DOI 10.1007/s10867-010-9201-9.

The definitive version is available at:

http://imammb.oxfordjournals.org/ 


\title{
Ecoepidemics overcoming the species-barrier and being subject to harvesting
}

\author{
S. Chaudhuri, A. Costamagna, E. Venturino \\ Dipartimento di Matematica "Giuseppe Peano" \\ Universita' di Torino, \\ via Carlo Alberto 10, 10123 Torino, Italy
}

\begin{abstract}
An ecoepidemiological model in which the disease can be transmitted from one population to another one is considered. Linear harvesting on all the populations is considered. By means of numerical simulations the role of the epidemiological parameters as well as that of harvesting are investigated. Some relevant consequences of harvesting on the system dynamics are discovered.
\end{abstract}

Keywords ecoepidemics, harvesting, predator-prey.

\section{Introduction}

We present in this paper the first ecoepidemiological model in which harvesting is considered for diseases that cross the species barrier. The underlying population model has also some kind of novelty, in the sense that we assume that the disease can spread from one species to the other one. Most of the ecoepidemic research of the past fifteen years has indeed been devoted to diseases that remain confined in one population only.

In the next Section we briefly outline mathematical epidemiology and population theory, with a short review of some contributions in the recent

*The first author has been supported by "MIUR Bando per borse a favore di giovani ricercatori indiani" during his visit at the University of Torino. 
literature on ecoepidemic systems. In Section 3 the basic ecoepidemic model with the disease crossing the species barrier is presented. The model includes an additional feature with respect to previous models that appeared in the literature, represented by linear harvesting on the prey and on the predators. In the following Section we analytically investigate its features. In Section 5 extensive simulations are performed, and the findings are reported, highlighting the role of various sets of parameters on the final outcome of the system. A final discussion of the results concludes the paper.

\section{Population Models}

To motivate the model that we will introduce later, we present in this section a quick review of the basic epidemiological and population systems, see ? for more references. These models, merged together, produce the ecoepidemic models. In all the models we consider, the parameters are assumed nonnegative unless otherwise specified.

\section{$2.1 \quad$ Epidemics}

Diseases have always affected humanity. With the advent of scientific means of fighting them also mathematical epidemiology has been developed to study diseases propagation, with the aim of controlling and eradicating them. This is important, because the basic problem to face in this context is represented by the newly infected individuals, that are in general able to spread the disease before showing its symptoms, i.e. before they are recognizable as infectives.

In the classical models a single population $N$ is assumed fixed in size and partitioned in infectivity classes. Assuming mass action incidence and disease recovery $\gamma$, with $S$ denoting susceptible and $I$ infectious individuals, $N=S+I$ the model reads

$$
\frac{d S}{d t}=-b I S+\gamma I, \quad \frac{d I}{d t}=b I S-\gamma I
$$

More realistic models contain also the class of the quarantined individuals $R$ at rate $\delta$. Using standard incidence, we have

$$
\frac{d S}{d t}=-\frac{b}{N} S I+\gamma R, \quad \frac{d I}{d t}=\frac{b}{N} S I-\delta I, \quad \frac{d R}{d t}=\delta I-\gamma R
$$


Other refinements allow for variable populations and study more complex phenomena, i.e. epidemic waves, ? and prophilactic measure, ?. In fact it is through the investigation of models in epidemiology with variable population sizes, ?? that ecoepidemiology started. Quarantine as a measure of control of the disease can also be introduced, ?. The fairly recent review ? still represents a good introduction into the topic.

\subsection{Demographic models}

A single population $P$ reproduces according to

$$
\frac{d P}{d t}=r(P) P
$$

where

$$
r(P)=r ; \quad r(P)=r\left(1-\frac{P}{K}\right) ; \quad r(P)=r \frac{P}{H+P},
$$

give respectively the Malthus model, the logistic or Holling type I model with linear reproduction rate, and the Michaelis-Menten or Holling type II dynamics, ?.

Devised about a century ago, ??, predator-prey models contain the predators $P$ and the prey $Q$; for instance

$$
\frac{d Q}{d t}=r Q\left(1-\frac{Q}{K}\right)-a Q P, \quad \frac{d P}{d t}=g P+a e Q P-b P^{2},
$$

contains logistic growth for the prey, a mass action type of interaction with the predators, conversion factor of captured prey into new predators $e$ and predators' intraspecific competition rate $b \neq 0$. Note that $g<0$ represents predators mortality, while $g>0$ indicates that other food sources are available to them. Generalizations include food chains, in which several trophic levels are accounted for, ??. The populations in the intermediate levels become predators of those at the lower level and prey for those on top of them. At the bottom we find the phytoplankton in the ocean and the vegetables on land, and at the top the large predators and ultimately man.

\subsection{Ecoepidemiological models}

These models merge a demographic model with an epidemic affecting at least one of the system's populations, ??. Specific diseases in an acquatic 
enviroment were considered in ?. Theoretical models with the underlying structure of the basic Lotka-Volterra model were introduced in ??. In ? the disease is assumed to affect the prey and in ? the model is extended with the predation term being modeled via a Holling-type II response function. In the first decade of this century a number of other papers has appeared, among which ???, mainly concentrating on epidemics spreading among the prey.

Also disease-affected predators have been the subject of recent research, ?. In ? a highly nonlinear predator-prey model is studied where prey host a disease that can be transmitted by contact, with transmission rate being frequency-dependent. Periodic attractors of the system's trajectories are discovered, which may be related to observed phenomena in nature such as sudden population bursts.

The invasion of a resident predator-prey system by an infectious disease with frequency-dependent transmission spreading within the predator population is considered in ?. The results are classified in terms of the reproduction numbers: the disease can dampen the cycles of the underlying demographic system, or alternatively, predators may become extinct. In other studies, however, the destabilizing role of the epidemic on the stability of the demographic model is emphasized, see for instance ????.

The Feline Immonodeficiency Virus (FIV) has been humanly-introduced into the cats on islands in order to prevent their hunting of the autoctonous birds. In ? it is found that it keeps their number in check, lowering their equilibrium value or the range of their oscillations, so that the prey can recover. Some differences are found to depend on the type of mixing considered. The predators population cannot be eradicated by mass action disease transmission, but they can with proportionate mixing.

Other types of population interactions have also been considered, competing ? and symbiotic, ??.

Research on diseases that are capable of affecting both populations simultaneously has resumed in more recent years, ??. The present contribution fits into this scheme by expanding ? with the introduction of populations harvesting.

In our system we consider the interactions based on a quadratic predatorprey population model. Both species are affected by the disease, which is able to overcome the species barrier. In addition, also vertical disease transmission is considered. Our main goal here is the study of how harvesting influences the system dynamics. 


\section{The proposed ecoepidemic model}

We consider a predator-prey ecosystem, in which both populations are diseaseaffected and the epidemics can be transmitted from one species to the other one by interaction. The driving reason prompting this investigation of such issues relies in the recent epidemics of wide interest in the media, such as avian influenza, ?, Severe Acute Respiratory Syndrome and the Swine flu epidemic originated in Mexico.

With respect to the two previous papers mentioned above, namely ??, here we consider also logistic reproduction of the infected prey. On the epidemiological side, the model accounts for infection of prey due to contact with infected predators and vertical transmission of the disease. But in ? predation is modeled via a Michaelis-Menten term, while here we use a simple mass action form. While predators in ? get the disease by feeding upon infected prey, here the predators' interaction with infected prey has a positive effect, avoiding predators starvation, and a negative one, when during the contact with the infected prey the sound predator possibly contracts the disease. Furthermore, predators mortality is linear in ?, while the logistic assumption makes it quadratic here. Note also that the simplifying assumption that a sound prey always escapes the attack of an infected predator, ?, is here removed allowing for a more general description of the interactions. Furthermore, as noted above, infection here trasmits vertically as well. In addition, the specific contribution of this model is contained in the harvesting terms.

The model reads

$$
\begin{aligned}
& \frac{d P(\tau)}{d \tau}=P\left(r_{1}-\frac{P+U}{\widetilde{K}}-\gamma U-\beta V-b_{1} Q-b_{3} V\right)-h P \\
& \frac{d U(\tau)}{d \tau}=U\left(r_{2}-\nu+\gamma P-\frac{P+U}{\widetilde{K}}-b_{2} Q-b_{4} V\right)+\beta P V-\ell U \\
& \frac{d Q(\tau)}{d \tau}=Q\left(-m-\alpha U-\eta V+e\left(b_{1} P+b_{2} U\right)\right)-\pi Q \\
& \frac{d V(\tau)}{d \tau}=V\left(-m-\mu+\eta Q+e\left(b_{3} P+b_{4} U\right)\right)+\alpha U Q-n V .
\end{aligned}
$$

Here $P$ represents the sound prey, $U$ the infected prey, $Q$ the sound predators and $V$ the infected predators. The model essentially differs from ? since it contains harvesting, represented by the last term in each equation. Therefore $h$ is the harvesting rate of sound prey, $\ell$ the one of infected prey, $\pi$ and $n$ 
respectively the removal rates by an external agent of healthy and diseased predators. We assume them to be different for more generality, although, in case of fisheries for instace, it would make sense to have them all equal. We now describe in detail the meaning of each equation.

The first equation describes the sound prey, reproducing logistically, at net growth rate $r_{1}$ and population pressure $\widetilde{K}^{-1}$, incorporating also the one of infected prey. Here the disease is assumed not to affect the prey intraspecific competition for resources. The third and fourth terms describe the infection process, by contact with an infected prey and an infected predator respectively. The last terms in the bracket instead account for predation, by both sound and infected predators at the rates $b_{1}$ and $b_{3}$. Here and in all the following equations, the final term represents linear harvesting. Note that we assume a very general situation, allowing for the possibility that a sound prey escapes from predation, but during the interaction with an infected predator maybe it gets wounded and in this way it catches the disease. In specific instances in which this possibility is not biologically feasible, the corresponding coefficient can be set to zero, to accomodate this case.

In the second equation we find the infected prey dynamics. Individuals enter into this class via the sound prey contacts with infected prey and predators at respective rates $\gamma$ and $\beta$ as stated above. We allow also for vertical trasmission of the disease at rate $r_{2}$ and disease mortality at rate $\nu$. Intraspecific competition leads to quadratic mortality which we assume with the same constant $\widetilde{K}^{-1}$ as for sound prey. Hunt by both sound and diseased predators at the rates $b_{2}$ and $b_{4}$ is modeled by the fourth and fifth terms in the bracket. We assume $b_{2}>b_{1}, b_{4}>b_{3}$ meaning that infected prey are more vulnerable than sound ones.

In absence of their prey, the third and fourth equations state that predators die at natural rate $m$ with additional disease-related mortality $\mu$, i.e. $P$ and $U$ represent the predators only food source. The conversion factor of captured prey into new predators is modeled by $e \leq 1$. Finally the disease incidences with contacts with infected predators and infected prey are respectively $\eta$ and $\alpha$. A new recruit among the infected predators occurs via contact with another infected predator, but also with interaction with an infected prey. This last situation is perhaps unlikely, but we still would like to make a general model allowing it. Specifically, it could occur if the predator comes into contact for instance with the ground contaminated by the infected prey. We would like our model to encompass these situations as well. Note also that in formulating the feeding process we state that the ingested prey 
does not cause harm to the capturing predator. With discussions with our biological colleagues, these assumptions can reasonably be thought to hold especially for the large predators of the African savannas. On the contrary, on the opposite scale at the plankton level, models for the poisonous action that toxic phytoplankton exerts on zooplankton feeding on it are well-known, ????. These indicate this phenomenon as one possible cause of the so-called red (or brown) tides. In this model however we explicitly exclude this effect.

\section{Analysis of the system}

In this section some of the mathematical details have been omitted, but the interested reader can find them in the Appendix.

\subsection{Boundedness}

Consider the total environmental population $S(\tau)=P+U+Q+V$. Letting $M=P+U, R=Q+V$, adding the equations (1) for a suitable $\theta$, with $m>\theta>0$ it follows

$S^{\prime}(\tau)=r_{1} P+r_{2} U-\mu U-m R-\frac{M^{2}}{\widetilde{K}}-(1-e) R\left(b_{1} P+b_{2} U\right) \leq\left(r_{1}+r_{2}+\theta\right) M-\frac{M^{2}}{\widetilde{K}}$.

Using Gronwall's inequality we find $0<S(t)<\left(1-e^{-\theta t}\right) W^{*}+S(0) e^{-\theta t}$ with $W^{*} \equiv \widetilde{L} \widetilde{K}\left(r_{1}+r_{2}+\theta\right)^{2}$ and $\widetilde{L}=(4 \theta)^{-1}$. Thus for $t \longrightarrow+\infty$, we have $S(t) \longrightarrow W^{*}>0$ and the system is therefore bounded.

\subsection{System's long term behavior}

Changing the variables $p(t)=\theta P(\tau), u(t)=\phi U(\tau), q(t)=\psi Q(\tau), v(t)=$ $\omega V(\tau), t=\tau \sigma$, where

$$
\sigma=m, \quad \phi=\frac{\gamma}{m}, \quad \omega=\frac{\beta}{m}, \quad \theta=\frac{e b_{1}}{m}, \quad \psi=\frac{b_{1}}{m}
$$

and letting

$$
\begin{aligned}
& A=\frac{\gamma}{e b_{1}}, \quad B=\frac{1}{\widetilde{K} \gamma}, \quad C=\frac{b_{3}}{\beta}, \quad D=\frac{b_{4}}{\beta}, \quad E=\frac{\alpha}{\gamma}, \quad F=\frac{\eta}{\beta} \\
& G=\frac{b_{3}}{b_{1}}, \quad X=\frac{e b_{2}}{\gamma}, \quad R_{1}=\frac{r_{1}}{m}, \quad R_{2}=\frac{r_{2}-\nu}{m}, \quad M=\frac{\mu}{m},
\end{aligned}
$$


we adimensionalize (1) to get

$$
\begin{aligned}
\frac{d p}{d t} & =p\left[R_{1}-A B p-B u-u-v-q-C v-H\right] \\
\frac{d u}{d t} & =u\left[R_{2}-A B p-B u+A p-A X q-D v-L\right]+A p v \\
\frac{d q}{d t} & =q[-1+p+X u-E u-F v-\Pi] \\
\frac{d v}{d t} & =v\left[-1-M+G p+\frac{D G}{A C} u+\frac{F G}{C} q-N\right]+\frac{E G}{C} u q,
\end{aligned}
$$

where

$$
H=\frac{h}{m}, \quad L=\frac{\ell}{m}, \quad \Pi=\frac{\pi}{m}, \quad N=\frac{n}{m} .
$$

\subsubsection{Equilibria}

We find the following boundary points.

The predator-prey free equilibrium is $Q^{(1)}=(0,0,0,0)$, the predator and infected prey-free or also sound prey-only equilibrium, $Q^{(2)}=\left(\frac{R_{1}-H}{A B}, 0,0,0\right)$, the disease free equilibrium $Q^{(3)}=\left(1+\Pi, 0, R_{1}-A B-H-A B \Pi, 0\right)$, the predator free equilibrium $Q^{(4)}=\left(p^{(4)}, u^{(4)}, 0,0\right)$, where

$$
\begin{aligned}
& p^{(4)}=\frac{1}{A}\left[B\left\{R_{1}+L-\left(R_{2}+H\right)\right\}-\left(R_{2}-L\right)\right] \\
& u^{(4)}=B\left\{R_{2}+H-\left(R_{1}+L\right)\right\}+\left(R_{1}-H\right),
\end{aligned}
$$

the sound predator and sound prey-free equilibrium $Q^{(5)}=\left(0, u^{(5)}, 0, v^{(5)}\right)$, where

$$
u^{(5)}=\frac{A C}{G D}(1+M+N), v^{(5)}=\frac{\left(R_{2}-L\right) G D-A B C(1+M+N)}{G D^{2}},
$$

the sound prey only-free equilibrium $Q^{(6)}=\left(0, u^{(6)}, q^{(6)}, v^{(6)}\right)$, with

$$
\begin{array}{r}
u^{(6)}=\frac{1+\Pi+F v_{(6)}}{X-E}, \\
q^{(6)}=\frac{\left(R_{2}-L\right)(X-E)-B(1+\Pi)-\{B F+D(X-E)\} v^{(6)}}{A X(X-E)}
\end{array}
$$


and where $v^{(6)}$ is a positive root of the quadratic equation

$$
\hat{A}_{h} v^{2}+\hat{B}_{h} v+\hat{C}_{h}=0
$$

whose coefficients (23) are given in the Appendix.

Further, the interior equilibrium is $E^{*}=\left(p^{*}, u^{*}, q^{*}, v^{*}\right)$, where $\left(u^{*}, v^{*}\right)$ is a point of intersection of the following two curves in the $u, v$ plane

$$
\begin{aligned}
& \widehat{A}_{1} u^{2}+2 \widehat{H}_{1} u v+\widehat{B}_{1} v^{2}+2 \widehat{G}_{1} u+2 \widehat{F}_{1} v=0, \\
& \widehat{A}_{2} u^{2}+2 \widehat{H}_{2} u v+\widehat{B}_{2} v^{2}+2 \widehat{G}_{2} u+2 \widehat{F}_{2} v=0,
\end{aligned}
$$

with $\left(p^{*}, q^{*}\right)$ given in terms of $u^{*}$ and $v^{*}$ as follows

$$
\begin{array}{r}
p^{*}=1+(E-X) u^{*}+F v^{*}, \\
q^{*}=\left(R_{1}-A B\right)+(A B X-A B E-B-1) u^{*}-(1+C+A B F) v^{*} .
\end{array}
$$

The relevant quantities (24), (25) appearing in these expressions are contained in the Appendix for the interested reader.

\subsubsection{Feasibility}

$Q^{(1)}$ always exists; $Q^{(2)}$ is feasible if and only if

$$
H<R_{1} \text {. }
$$

The feasibility condition of $Q^{(3)}$ is

$$
H<R_{1}-A B(1+\Pi) .
$$

The predator free equilibrium $Q^{(4)}$ is feasible if either one of the following conditions holds

(a) $R_{1}-\frac{B\left(R_{2}-L\right)}{B-1}<H<R_{1}-\frac{\left(R_{2}-L\right)(1+B)}{B}$, when $B>1$ or

(b) $H<R_{1}-\frac{\left(R_{2}-L\right)(1+B)}{B}$, when $B \leq 1$.

$Q^{(5)}$, in turn, is feasible if and only if

$$
L<R_{2}-\frac{A B C(1+M+N)}{G D} .
$$


The following conditions ensure the feasibility of $Q^{(6)}$

$$
\text { (a) } X>E \quad \text { (b) } 0<v^{(6)}<\frac{\left(R_{2}-L\right)(X-E)-B(1+\Pi)}{B F+D(X-E)} \text {. }
$$

The interior equilibrium has been thoroughly analysed in ?. We summarize here only the basic facts. It depends on the intersection of conic sections giving the point $\left(p^{*}, q^{*}\right)$.

Now if either one of the conditions

$$
\text { (a) } \widehat{G}_{1}<0, \widehat{A}_{1}>0, \widehat{F}_{1}>0 \quad(b) \widehat{G}_{1}>0, \widehat{A}_{1}<0, \widehat{F}_{1}<0
$$

holds, or alternatively if either one of the conditions

$$
\text { (a) } \widehat{G}_{2}>0, \widehat{A}_{2}<0, \widehat{F}_{2}>0, \quad(b) \widehat{G}_{2}>0, \widehat{A}_{2}>0, \widehat{F}_{2}<0
$$

is verified, sufficient conditions for feasibility of $E^{*}$ are for $i=1,2$

$$
\widehat{v}_{1}<v_{*}<\widehat{v}_{2}, \quad \widehat{D}_{1 i} \neq 0, \quad \frac{\widehat{G}_{1}}{\widehat{A}_{1}} \leq \frac{\widehat{G}_{2}}{\widehat{A}_{2}} .
$$

The quantities appearing in (16) are listed in the Appendix, see (26), (27).

\subsubsection{Stability}

In view of the eigenvalues of the Jacobian matrix (28) evaluated at $Q^{(1)}$, the origin is locally asymptotically stable if the following conditions are satisfied

$$
H>R_{1}, \quad L>R_{2}
$$

The conditions for local asymptotic stability of $Q^{(2)}$, see (29), are explicitly

$$
\begin{array}{r}
L>\frac{B R_{2}-\left(R_{1}-H\right)(B-1)}{B}, \\
\Pi>\frac{\left(R_{1}-H\right)-A B}{A B}, \quad N>\frac{G\left(R_{1}-H\right)-A B(1+M)}{A B} .
\end{array}
$$

For the disease-free equilibrium $Q^{(3)}$ the Routh-Hurwitz conditions, see (31), hold if

$$
\begin{gathered}
L>R_{2}+(A-A B) p^{(3)}-A X q^{(3)} \\
N>-(1+M)+G p^{(3)}+\frac{G F}{C} q^{(3)}, \quad A E G p^{(3)} q^{(3)}<C \hat{X}_{h} \hat{Y}_{h} .
\end{gathered}
$$


For stability of the predator-free equilibrium $Q^{(4)}$, see (32) and (33), we need

$$
\begin{array}{r}
\Pi>\Pi^{i v}, \quad N>N^{i v}, \quad B \leq 1, \\
\Pi^{i v}=-1+p^{(4)}+X u^{(4)}-E u^{(4)}, \\
N^{i v}=-(1+M)+G p^{(4)}+G D A^{-1} C^{-1} u^{(4)} .
\end{array}
$$

For $Q^{(5)}$ the stability conditions are instead, see (34) and (35),

$$
\begin{array}{r}
H>H^{v}, \quad H^{v}=R_{1}-(B+1) u^{(5)}-(1+C) v^{(5)}, \\
\Pi>\Pi^{v}, \quad \Pi^{v}=-1+(X-E) u^{(5)}-F v^{(5)} .
\end{array}
$$

Finally those for the equilibrium $Q^{(6)}$ are

$$
H>H^{v i}, \quad X<X_{h}^{v i}, \quad e<e_{h}^{v i}
$$

where

$$
\begin{aligned}
H^{v i} & =R_{1}-(B+1) u^{(6)}-(1+C) v^{(6)}-q^{(6)} \\
X_{h}^{v i} & =\frac{B\left(G F v^{(6)}+G E u^{(6)}\right)}{G D v^{(6)}+A E G q^{(6)}}, \quad e_{h}^{v i}=\frac{D(X-E)}{E F A} \frac{v^{(6)}}{q^{(6)}} .
\end{aligned}
$$

For the coexistence equilibrium point $E^{*}=\left(p_{*}, u_{*}, q_{*}, v_{*}\right)$ the characteristic equation is a monic quartic, similar to the one of ?, which is far too complicated to analize numerically. So we will investigate numerically the behavior of the solution.

In absence of harvesting the system has been shown to possess no periodic orbits, ?. This result remains valid in presence of harvesting because the partial derivatives needed by the Dulac's criterion used in the proof remain the same also in this case.

\section{Numerical Experiments}

To run simulations, we choose the parameter values for the original system (1) already used in our former investigation, ?, which were based on informations gathered from the literature. We report them here for the convenience of the reader: $r_{1}=3, \widetilde{K}=15, r_{2}=1.5, \nu=0.24, m=0.09, e=0.4, \mu=0.08$, $\gamma=0.4, \beta=0.3, \alpha=0.002, \eta=0.0034, b_{1}=0.06, b_{2}=0.08, b_{3}=0.02$, 
$b_{4}=0.04, h=0.015, l=0.03, \pi=0.01, n=0.02$. From these we obtain the following values of the parameters of the adimensionalized system (2): $A=16.6667 B=0.1667, C=0.0667, D=0.1333, E=0.0050, F=0.0113$, $G=0.3333, X=0.0800, R_{1}=33.3333, R_{2}=14, M=0.8889, H=0.1667$, $L=0.3333, \Pi=0.1111, N=0.2222$.

These satisfy the sufficient conditions (14), (15), and the feasibility condition (16) of $\left(u^{*}, v^{*}\right)$. From the relations $(7)$ and (8) we have $\left(p^{*}, q^{*}\right)=$ $(0.35,14.14)$, giving the coexistence equilibrium $(0.35,10.85,14.14,5.05)$.

\subsection{Role of population harvesting}

First we will fix the harvesting parameters, namely $l, \pi$ and $n$ at the values 0.03, 0.01 and 0.02 respectively. We allow to vary the sound prey harvesting coefficient $h$ in its permissible range, $0 \leq h \leq l$ since harvesting of infected prey, as they are weaker, cannot be smaller than the sound ones. In the range $0 \leq h \leq 0.03$ all the populations persistently coexist at the interior equilibrium, see for instance Fig. 1 for $h=0.025$.

Keeping the other parameters fixed at the values $h=0.015, \pi=0.01$ and $n=0.02$, we investigate the effect of varying the infected prey harvesting, with the restriction $l \geq 0.015$. In a wide range, $0.015 \leq l<4.88$, all populations show a stable dynamics towards the coexistence equilibrium. But beyond this range the infected prey are wiped out of the system, see Fig. 2 for $l=5.00$. 

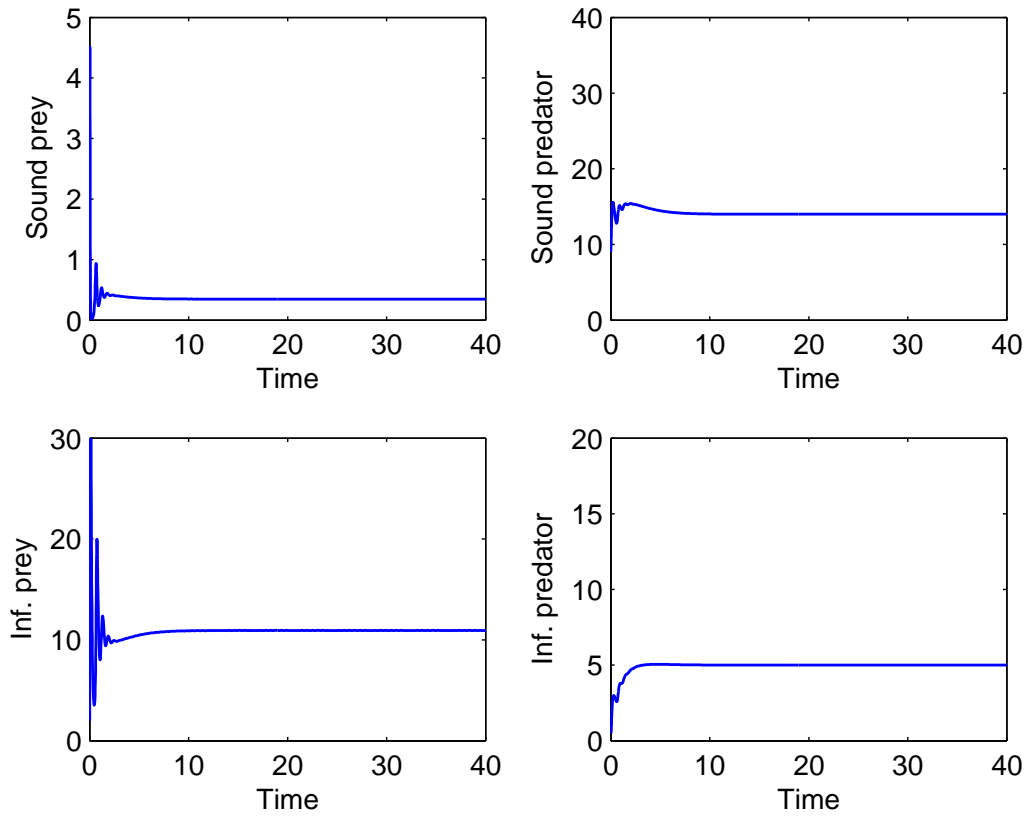

Figure 1: Evolution of the system populations (2) towards coexistence is obtained for the following parameter values: $A=16.6667, B=0.1667$, $C=0.0667, D=0.1333, E=0.0050, F=0.0113, G=0.3333, X=0.0800$, $R_{1}=33.3333, R_{2}=14, M=0.8889, H=0.2778, L=0.3333, \Pi=0.1111$, $N=0.2222$. Top row: healthy populations, left column: prey. 

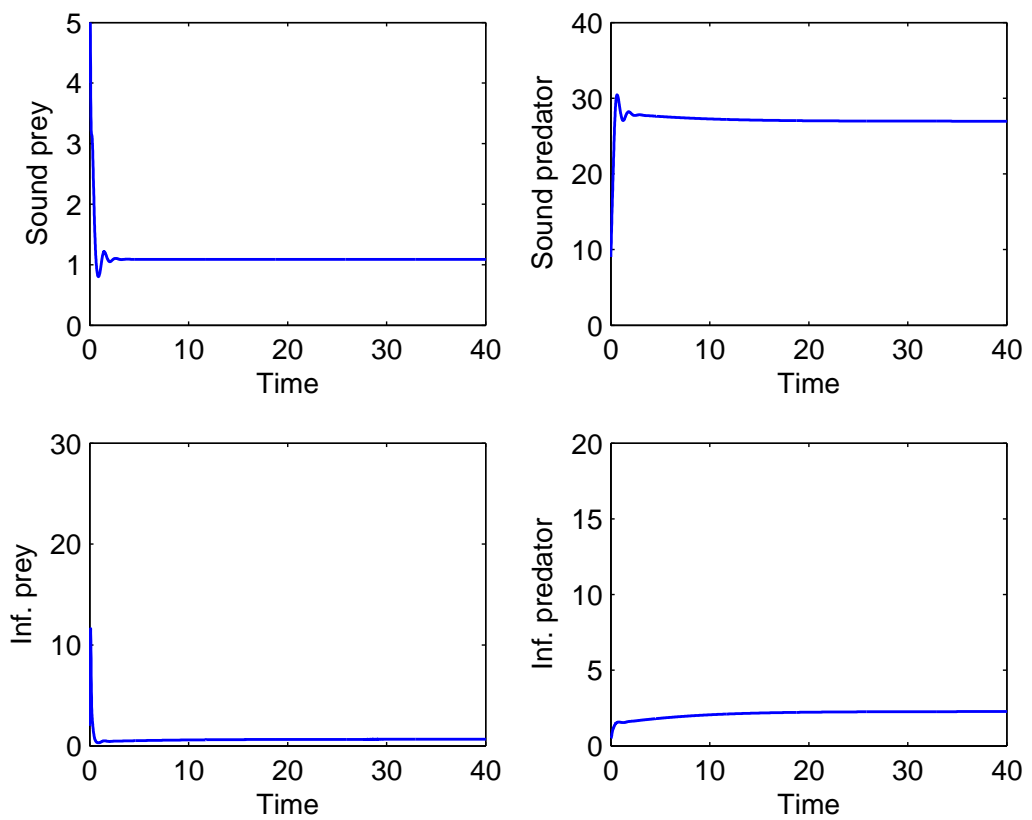

Figure 2: The prey are wiped out from the system (2) when: $A=16.6667$, $B=0.1667, C=0.0667, D=0.1333, E=0.0050, F=0.0113, G=0.3333$, $X=0.0800, R_{1}=33.3333, R_{2}=14, M=0.8889, H=0.1667, L=55.5556$, $\Pi=0.1111, N=0.2222$. Top row: healthy populations, left column: prey. 

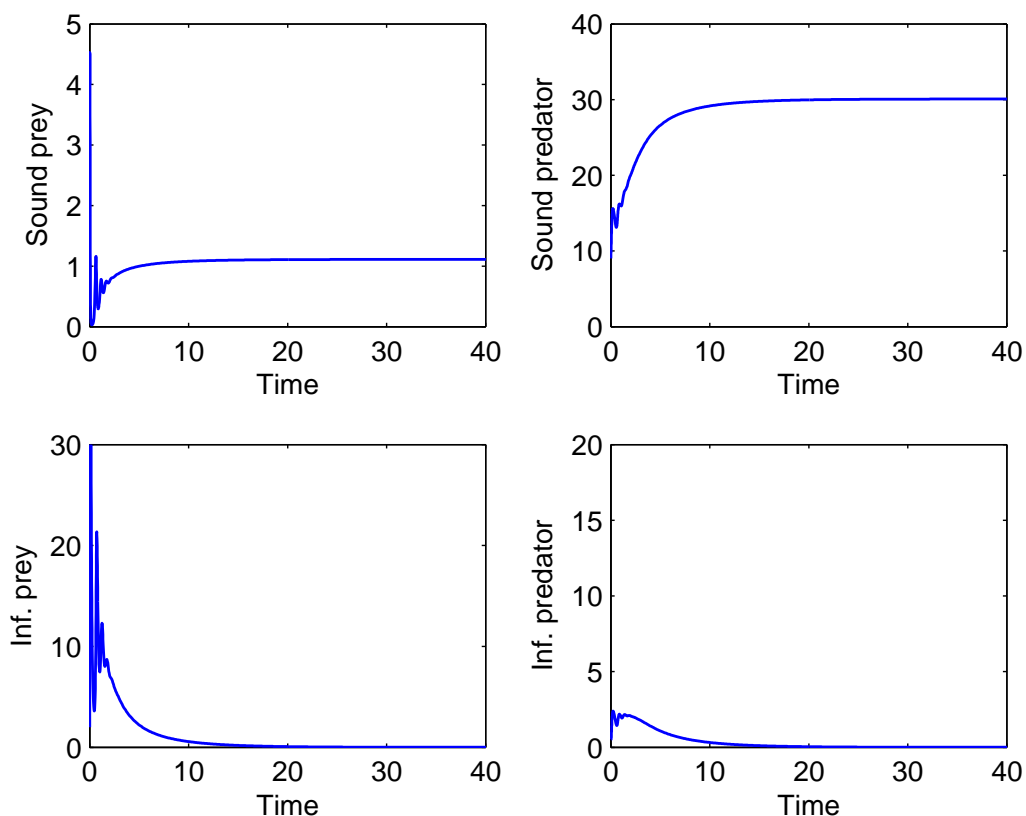

Figure 3: The disease is eradicated from the system (2) for: $A=16.6667$, $B=0.1667, C=0.0667, D=0.1333, E=0.0050, F=0.0113, G=0.3333$, $X=0.0800, R_{1}=33.3333, R_{2}=14, M=0.8889, H=0.1667, L=0.3333$, $\Pi=0.1111, N=1.6667$. Top row: healthy populations, left column: prey.

For a similar reason as in the case of prey, the sound predator harvesting coefficient is also bounded i.e, $\pi \leq n$. Hence for the fixed values $h=0.015$, $l=0.03, n=0.02$, in the interval $0 \leq \pi \leq 0.02$ all the populations show a stable dynamics around the interior equilibrium.

Instead, the harvesting coefficient of the infected predators $n$ is bounded below by $\pi$, for the reason given earlier in the case of infected prey. If we choose the parameter values as $h=0.015, l=0.03, \pi=0.01$, in the small range $0.01 \leq n<0.13$ all the populations coexists in a stable fashion. But when $n \geq 0.13$ both the infected prey and predators are removed from the system, for example see Fig. 3 for $n=0.15$. Therefore a small increase in infected predator harvesting can eradicate the disease from the system. For the convenience of the reader we present a summary of our numerical findings in Table 1. 


\begin{tabular}{|c|c|c|}
\hline $\begin{array}{c}\text { Fixed Parameters } \\
\text { and their value }\end{array}$ & $\begin{array}{c}\text { Parameter varied } \\
\text { in a range }\end{array}$ & Dynamics \\
\hline $\begin{array}{c}l=0.03, \pi=0.01, \\
n=0.02\end{array}$ & $0 \leq h \leq 0.03$ & APC \\
$h=0.015, \pi=0.01$, & $0.015 \leq l<4.88$ & APC \\
$n=0.02$ & $l \geq 4.88$ & DFP \\
& $0 \leq \pi \leq 0.02$ & APC \\
$h=0.015, l=0.03$, & & \\
$n=0.02$ & & APC \\
$h=0.015, l=0.03$, & $0.01 \leq n<0.13$ & DFS \\
$\pi=0.01$ & $n \geq 0.13$ & \\
\hline
\end{tabular}

Table 1: The abbreviations are as follows: APC for "all populations coexist"; DFP for disease-free prey; DFS for disease-free system. The Table contains the simulation results obtained by varying the harvesting rates. The other parameters are: $r_{1}=3, \widetilde{K}=15, \gamma=0.40, \beta=0.30 b_{1}=0.06, b_{3}=0.02$, $r_{2}=1.5, \nu=0.24, b_{2}=0.08, b_{4}=0.04, m=0.09, \alpha=0.0020, \eta=0.0034$, $e=0.4, \mu=0.08$

\subsection{Role of other parameters in presence of harvesting}

\subsubsection{Effect of epidemiological parameters}

Now we study how the epidemiological parameters affect the dynamics of the system populations in presence of harvesting. In the numerical simulations that follow the harvesting parameters are fixed at the values $h=0.015$, $l=0.03, \pi=0.01$ and $n=0.02$ with the predation coefficients $b_{1}=0.06$, $b_{3}=0.02, b_{2}=0.08$ and $b_{4}=0.04$.

At first we let the intraspecific disease transmission coefficient $\gamma$ vary in the prey population by keeping the other ones fixed, $\beta=0.3000, \alpha=0.0020$ and $\eta=0.0034$. In the region $0.051 \leq \gamma<0.49$ all populations coexist, but for lower values, namely $0<\gamma \leq 0.051$ the prey become disease free. Instead for larger values, specifically $\gamma \geq 0.49$, the sound prey become extinct. Fig. 4 shows the results for $\gamma=0.55$.

The other mechanism for which the prey can get infected is by contact with the infected predators via the disease transmission coefficient $\beta$. For 
the fixed parameter values $\gamma=0.4, \alpha=0.0020, \eta=0.0034$, if $\beta$ varies in the interval $0<\beta \leq 0.018$ then the system is disease-free, see Fig. 5 for $\beta=$ 0.005 . In the small range $0.019 \leq \beta<0.021$ only the prey become diseasefree, see Fig. 6 for $\beta=0.0195$. For further increase in $\beta$, in particular when $\beta \geq 0.021$, the disease invades both the prey and the predator populations.

We consider next the interspecific disease transmission $\alpha$ for which the predators are infected by the prey. To this end we fix the remaining disease transmission coefficients as follows $\gamma=0.04, \beta=0.3, \eta=0.0034$. All the populations of the system coexist in the interval $0.0002 \leq \alpha<.0031$.
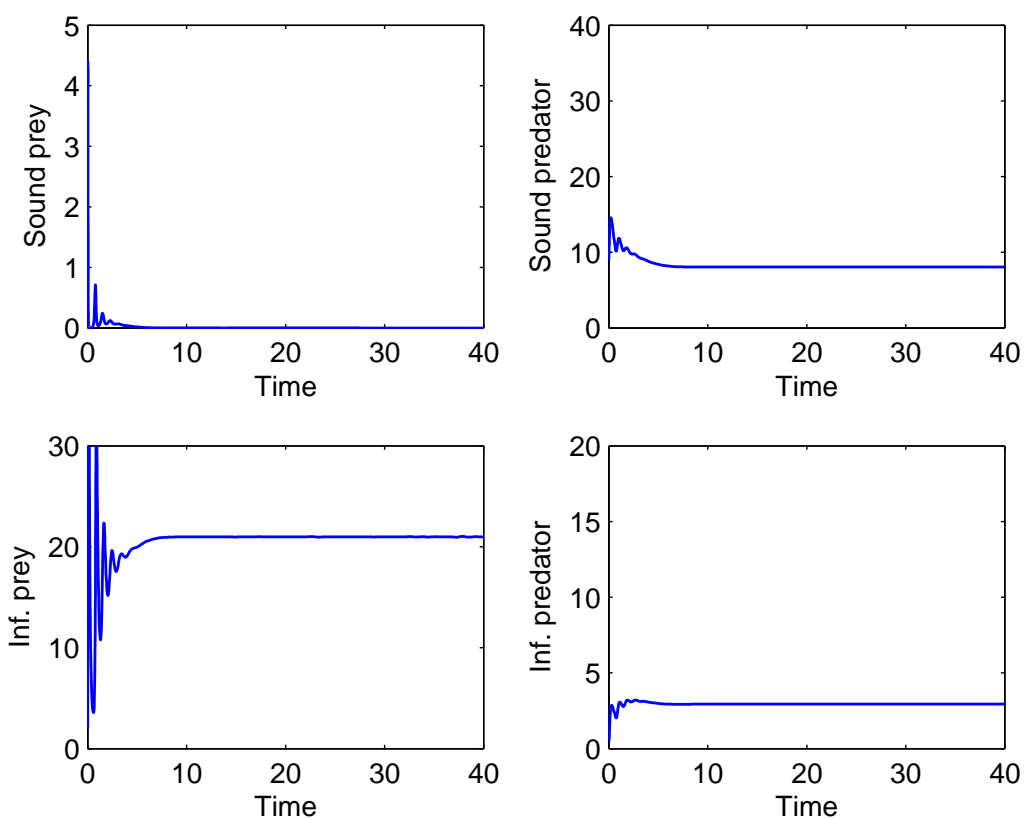

Figure 4: Extinction of the healthy prey from the system (2) is obtained for: $A=22.9167, B=0.1212, C=0.0667, D=0.1333, E=0.0036, F=0.0113$, $G=0.3333, X=0.0582, R_{1}=33.3333, R_{2}=14, M=0.8889, H=0.1667$, $L=0.3333, \Pi=0.1111, N=1.6667$. Top row: healthy populations, left column: prey. 

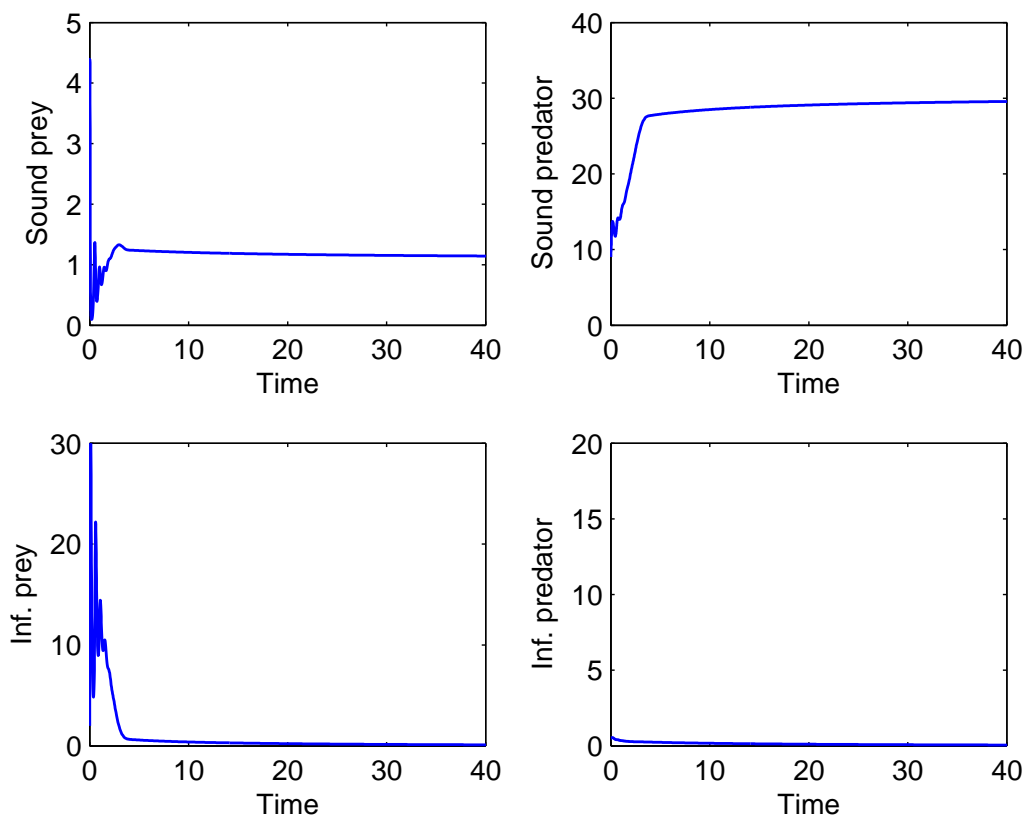

Figure 5: The system (2) becomes disease-free for: $A=16.6667, B=0.1667$, $C=4, D=8, E=0.0050, F=0.6800, G=0.3333, X=0.0800, R_{1}=$ 33.3333, $R_{2}=14, M=0.8889, H=0.1667, L=0.3333, \Pi=0.1111$, $N=0.2222$. Top row: healthy populations, left column: prey. 

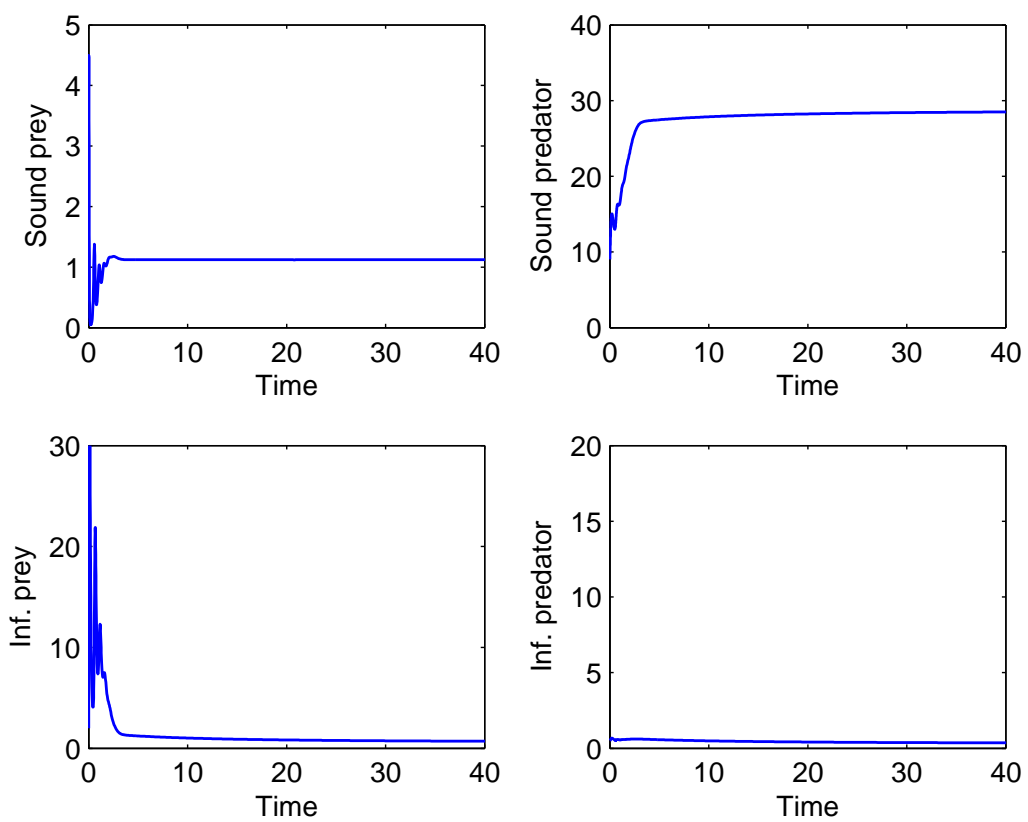

Figure 6: The disease is eradicated only from the prey for: $A=16.6667$, $B=0.1667, C=1.0256, D=2.0513, E=0.0050, F=0.1744, G=0.3333$, $X=0.0800, R_{1}=33.3333, R_{2}=14, M=0.8889, H=0.1667, L=0.3333$, $\Pi=0.1111, N=0.2222$. Top row: healthy populations, left column: prey.

However, if we take a value of $\alpha$ below 0.0002 , then the disease is eradicated from the system. When $0.0031 \leq \alpha<.02$ we observe extinction of the sound prey, see Fig. 7 for $\alpha=0.009$. A further extreme increase with $\alpha \geq 0.02$ wipes out both the sound prey and predators, see Fig. 8 for $\alpha=0.09$.

For the predator population $\eta$ denotes the intraspecific disease transmission coefficient. We observe its effect on the system when the other parameters namely, $\alpha, \beta, \gamma$ are fixed at the values $0.002,0.3$ and 0.4 respectively. When $\eta$ varies in $0.0011 \leq \eta \leq .00054$ all populations go to the coexistence equilibrium. If $\eta$ varies in the small interval $0<\eta<0.0011$, the system becomes disease-free. Instead, in $0.0055 \leq \eta<0.019$ the sound prey are wiped out. For an extreme increase, namely when $\eta \geq 0.019$, both the sound prey and predators disappear from the system. Table 2 contains a summary of the above results. 

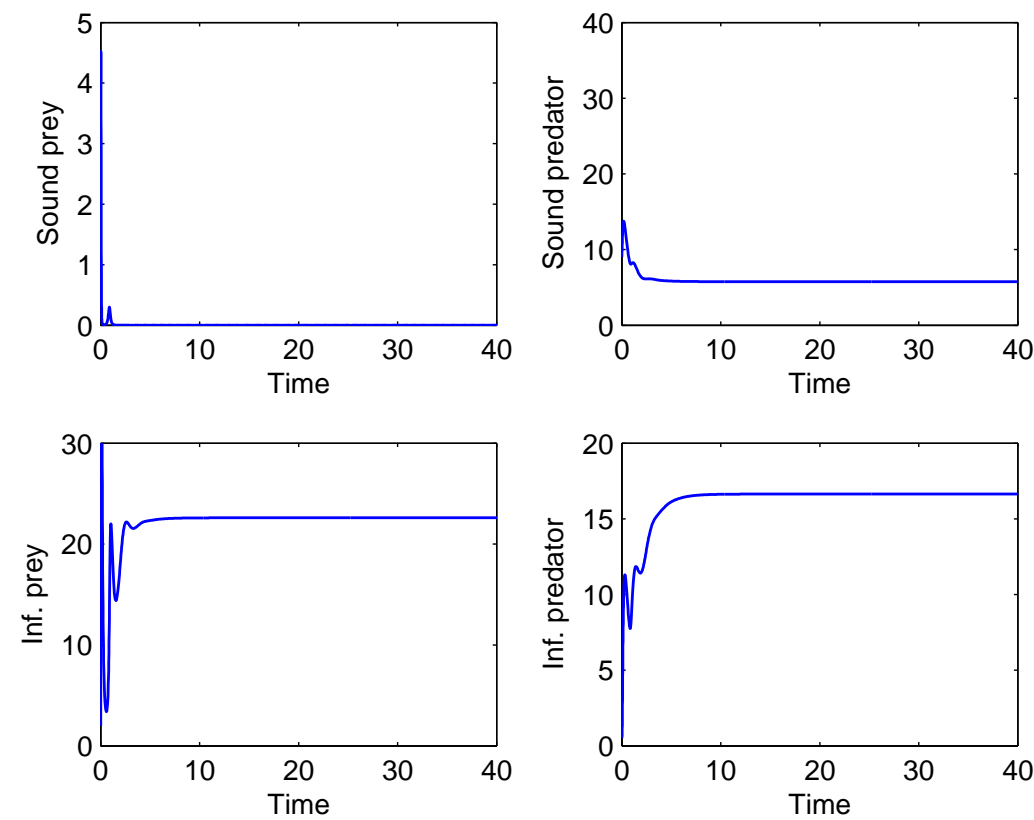

Figure 7: The healthy prey do not survive in the system (2) for: $A=16.6667$, $B=0.1667, C=0.0667, D=0.1333, E=0.0225, F=0.0113, G=0.3333$, $X=0.0800, R_{1}=33.3333, R_{2}=14, M=0.8889, H=0.1667, L=0.3333$, $\Pi=0.1111, N=0.2222$. Top row: healthy populations, left column: prey. 

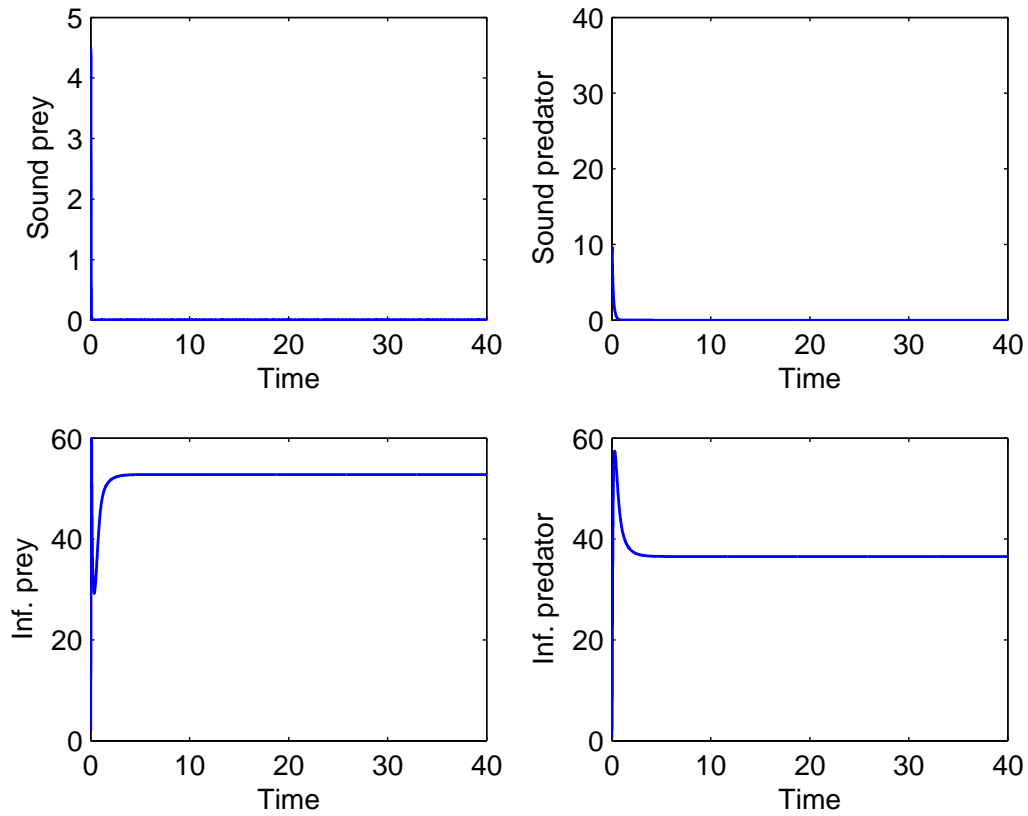

Figure 8: Both healthy populations in the system (2) do not survive for: $A=16.6667, B=0.1667, C=0.0667, D=0.1333, E=0.2250, F=0.0113$, $G=0.3333, X=0.0800, R_{1}=33.3333, R_{2}=14, M=0.8889, H=0.1667$, $L=0.3333, \Pi=0.1111, N=0.2222$. Top row: healthy populations, left column: prey. 


\begin{tabular}{|c|c|c|}
\hline $\begin{array}{c}\text { Fixed Parameters } \\
\text { and their value }\end{array}$ & $\begin{array}{c}\text { Parameter varied } \\
\text { in a range }\end{array}$ & Dynamics \\
\hline$\beta=0.3000$, & $0<\gamma<0.051$ & DFP \\
$\alpha=0.0020$, & $0.051 \leq \gamma<0.49$ & APC \\
$\eta=0.0034$ & $\gamma \geq 0.49$ & SPE \\
& & \\
$\gamma=0.4$, & $0<\beta<0.019$ & DFS \\
$\alpha=0.002$, & $0.019 \leq \beta<0.021$ & DFP \\
$\eta=0.0034$ & $\beta \geq 0.021$ & APC \\
& $0<\alpha<0.0002$ & DFS \\
$\gamma=0.4$, & $0.0002 \leq \alpha<0.0031$ & APC \\
$\beta=0.3$, & $0.0031 \leq \alpha<0.02$ & SPE \\
$\eta=0.0034$ & $\alpha \geq 0.02$ & SPPE \\
& $0<\eta<0.0011$ & DFS \\
$\gamma=0.4$ & $.0011 \leq \eta<0.0055$ & APC \\
$\beta=0.3$ & $.0055 \leq \eta<0.019$ & SPE \\
$\alpha=0.002$ & $\eta \geq 0.019$ & SPPE \\
\hline
\end{tabular}

Table 2: The abbreviations are as follows: APC for "all populations coexist"; DFP for disease-free prey; DFS for "disease-free system"; SPE for sound prey extinction; SPPE for sound prey and predators extinction. Here the results of the simulation obtained by varying the epidemiological coefficients are reported. The remaining parameters have the values: $r_{1}=3, \widetilde{K}=15$, $\gamma=0.40, \beta=0.30 b_{1}=0.06, b_{3}=0.02, r_{2}=1.5, \nu=0.24, b_{2}=0.08$, $b_{4}=0.04, m=0.09, \alpha=0.0020, \eta=0.0034, e=0.4, \mu=0.08, h=0.015$, $l=0.03, \pi=0.01, n=0.02$ 


\subsubsection{Effect of predation coefficients}

To simulate system dynamics against the predation rate we fix the epidemiological parameters at the values $\alpha=0.002, \beta=0.3, \gamma=0.4, \eta=0.0034$ while the harvesting parameters are chosen as follows $h=0.015, l=0.03$, $\pi=0.01$ and $n=0.02$.

The predation rate on sound prey by sound predators $b_{1}$ is bounded below by the corresponding rate of infected predators, i.e. $b_{1}>b_{3}$, because infected predators are less active in predation. We choose the remaining predation coefficients as $b_{3}=0.02, b_{2}=0.08$ and $b_{4}=0.04$. In the small interval $0.02<b_{1}<0.088$ all the populations coexist. Instead, beyond this interval i.e., for $b_{1} \geq 0.088$, the sound prey become extinct.

Predation of infected predators on sound prey must satisfy the same above restriction, $b_{3}<b_{1}$. Hence variation of $b_{3}$ will be restricted in $0<b_{3}<0.06$ once we have the other parameters as follows $b_{1}=0.06, b_{2}=0.08$ and $b_{4}=0.04$. In this range all the populations coexist.

Now we observe the effect of the predation rate on the infected prey by the sound predator $b_{2}$ when the other parameters are $b_{1}=0.06, b_{3}=0.02$ and $b_{4}=0.04$. Obviously we take $b_{2} \geq b_{1}=0.06$ because sound prey are more difficult to catch than infected ones. All populations coexist in the interval $0.06<b_{2}<0.19$, the prey become disease-free when $0.19 \leq b_{2}<0.57$. For extreme values of the predation rate both populations become disease-free, namely when $b_{2} \geq 0.57$.

Observe that clearly the predation rate on infected prey by infected predators is bounded. We have $0.02<b_{4}<0.080$ when the other parameters are taken as $b_{1}=0.06, b_{3}=0.02, b_{2}=0.08$. In $0.02<b_{4}<0.076$ all the populations go to the feasible interior equilibrium whereas in $0.076 \leq b_{4} \leq 0.080$ only the sound prey becomes extinct. In Table 3 these results are summarized.

\section{Discussion}

In the numerical simulations we always let the parameters vary in their permissible interval range.

Sound prey harvesting leads unquestionably to the stable coexistence equilibrium of all populations, Fig. 1. For infected prey the situation changes, since harvesting leads to a stable dynamics of all the populations when the 


\begin{tabular}{|c|c|c|}
\hline $\begin{array}{c}\text { Fixed Parameters } \\
\text { and their value }\end{array}$ & $\begin{array}{c}\text { Parameter varied } \\
\text { in a range }\end{array}$ & Dynamics \\
\hline$b_{3}=0.02, b_{2}=0.08, b_{4}=0.04$ & $0.02<b_{1}<0.088$ & APC \\
$b_{1} \geq 0.088$ & SPE \\
$b_{1}=0.06, b_{2}=0.08, b_{4}=0.04$ & $0<b_{3}<0.06$ & APC \\
$b_{1}=0.06, b_{3}=0.02, b_{4}=0.04$ & $0.06<b_{2}<0.19$ & APC \\
& $0.19 \leq b_{2}<0.57$ & DFS \\
& $b_{2} \geq 0.57$ & DFS \\
$b_{1}=0.06, b_{3}=0.02, b_{2}=0.08$ & $0.02<b_{4}<0.076$ & APC \\
& $0.076 \leq b_{4} \leq 0.080$ & SPE \\
\hline
\end{tabular}

Table 3: The abbreviations are as follows: APC for "all populations coexist"; DFS for "disease-free system"; SPE for "sound prey extinction". The simulation results are listed as function of the predation coefficients. The other fixed parameters are: $r_{1}=3, \widetilde{K}=15, \gamma=0.40, \beta=0.30 r_{2}=1.5$, $\nu=0.24, m=0.09, \alpha=0.0020, \eta=0.0034, e=0.4, \mu=0.08, h=0.015$, $l=0.03, \pi=0.01, n=0.02$ 
harvesting coefficient of infected prey falls below a critical value, while above it the prey becomes instead disease-free, Fig. 2.

As in the case of sound prey harvesting, no matter how the sound predator harvesting coefficient varies, the system always goes to the interior equilibrium point.

Harvesting of infected prey with rate below some critical value implies that all populations show a stable dynamics towards the coexistence equilibrium, but beyond this value the system becomes disease-free, as both the infected prey and predators are wiped out, Fig. 3.

When the intraspecific disease transmission coefficient $\gamma$ is below a threshold, the prey population becomes disease-free. Instead when it crosses another critical value, the sound prey become extinct, Fig. 4. When harvesting falls in the range between these values, all the populations stably coexist.

The other disease contact rate in the prey population is $\beta$ for the disease coming from interactions with infected predators. The system becomes disease-free when this rate is less than some critical value, but if it exceeds another threshold, then all populations go to the feasible interior equilibrium. Note that the difference between these critical values is very small; within this narrow range, the prey becomes disease-free, Fig. 6.

The interspecific disease transmission for predators $\alpha$ makes the system disease free when it is small but lies below some critical value. When the latter is crossed, within a certain interval, the system shows a stable coexistence dynamics. An increase of $\alpha$ beyond the upper endpoint of this interval, leads to the situation in which all populations coexist persistently. This holds true in a second interval. When $\alpha$ crosses its upper bound, and varies in another interval, the sound prey become extinct, Fig. 7. An extreme increase of $\alpha$ beyond this range wipes out both the sound prey and predators from the system, Fig. 8.

The intraspecific disease transmission coefficient $\eta$ among the predator population shows the same behavior as the one of interspecific disease transmission $\alpha$ except that there are changes in the thresholds and consequently in the ranges.

Sound prey become extinct from the coexistence state when the predation of sound predator upon the sound prey, $b_{1}$, exceeds some critical value whereas below this critical value all populations coexist.

On biological grounds we formulate the assumption that $b_{3}<b_{1}$, due to the fact that infected predators are less active in predation of sound prey than the sound predators are. Allowing the predation rate $b_{3}$ of infected 
predators on sound prey to vary within the above constraint, we find that all the populations coexist persistently.

Sound predators hunt more easily the infected prey since the latter are less able than the sound prey to avoid the predator's catching. Thus we impose the restriction $b_{2}>b_{1}$. All the populations coexist when the predation rate of infected prey by the sound predators lies below a certain value, but in another interval above it, the prey become disease-free. For extremely high predation rates, the disease gets eradicated from the system.

The predation rate of infected prey by infected predators is $b_{4}$. Now, infected predators are indeed more successful to predate infected prey than the sound ones while the infected predators are less able to hunt the prey than the sound ones. Hence, we have the natural restriction $b_{3}<b_{4}<b_{2}$. For a wide range of $b_{4}$ all populations show a stable dynamics, but above it, there is a small interval where the sound prey become extinct i.e., all prey get the infection, see the last row of Table 3.

Now in what follows we investigate the difference that the presence of harvesting entails on the undisturbed system dynamics.

In ? it has been found that in absence of harvesting the prey cannot be freed from the disease. This occurs for low values of the intraspecific disease transmission rate among the prey population, $\gamma$. Instead, here our findings reveal that in presence of harvesting the prey become disease-free.

Again in presence of harvesting, when the prey interspecific contact rate $\beta$ assumes low values, in a very narrow range, the system shows a disease-free state. For a small increase above this range the prey only become diseasefree. But in ? it has been found that in absence of harvesting the system settles neither to the disease-free nor to the prey-only disease-free state.

Disease spreading in the predator population due to infected prey is represented by the coefficient $\alpha$. In presence of harvesting the system becomes disease-free for small values of $\alpha$ within a narrow range. But in absence of harvesting, ?, the presence of a small number of infected prey makes the crossing of the species barrier possible for the disease, so that the epidemic invades the predator population, leading to the coexistence of all populations in the ecoepidemic system.

Harvesting however does not significantly affect the outcome of the disease spreading among the predators, when it is caused by intraspecific interactions, at rate $\eta$.

By comparing the Table 3 in ? and the Table 3 here, all the predation coefficients show the same effects on the qualitative dynamics of the system 
both in absence of harvesting and in its presence. The only exception is the predation rate of infected prey by the sound predators $b_{2}$. In fact, we have numerically found that in presence of harvesting a high predation rate of infected prey by the sound predators can make the system disease-free, but in ? a high predation rate without harvesting can remove the disease just from the prey population.

Acknowledgments: The authors thank the referees for their valuable comments and the biologist colleague Prof. Guido Badino for a very useful discussion on the matter.

\section{Appendix}

The coefficients of the quadratic (4) are

$$
\begin{aligned}
\hat{A}_{h}= & B F^{2} G X, \quad \hat{C}_{h}=G E(1+\Pi)\left[B(1+\Pi)-(X-E)\left(R_{2}-L\right)\right] \\
\hat{B}_{h}= & B F G X+B F G \Pi X-D G X^{2}+F G L X^{2}-D G \Pi X^{2}+A C X^{3} \\
& +A C M X^{3}+A C N X^{3}-B F G \Pi E+2 D G X E-2 F G L X E \\
& -2 A C X^{2} E-2 A C M X^{2} E-2 A C N X^{2} E+B F E G+F L X E G \\
& +D \Pi X E G+F G L E^{2}+A C X E^{2}+A C N X E^{2}-D E^{2} G \\
& -F L E^{2} G-D \Pi G E^{2}-F G X^{2} R_{2}+F G X E R_{2}+D G \Pi X E \\
& +A C M X E^{2}+2 B F \Pi E G .
\end{aligned}
$$

The coefficients of the quartics (5)-(6) are

$$
\begin{aligned}
& \widehat{G}_{1}=G_{1}+\frac{1}{2}[A X H-L-A \Pi(B-1-A B X)], \quad \widehat{A}_{2}=A_{2}, \quad \widehat{B}_{2}=B_{2}, \\
& \widehat{G}_{2}=G_{2}-\frac{A E G}{2}(H+A B \Pi), \quad \widehat{F}_{1}=F_{1}+\frac{1}{2} A \Pi, \quad \widehat{H}_{2}=H_{2}, \quad \widehat{H}_{1}=H_{1}, \\
& \widehat{F}_{2}=F_{2}+\frac{A}{2}[C G \Pi-F G(A B \Pi+H)-C N], \quad \widehat{A}_{1}=A_{1}, \quad \widehat{B}_{1}=B_{1},(24)
\end{aligned}
$$

where

$$
\begin{aligned}
A_{1} & =(A-A B)(E-X)-B-A X(A B X-A B E-B-1), B_{1}=F A \\
H_{1} & =\frac{1}{2}[A F(1-B)-D+A X(1+C+A B F)+(E-X) A], \\
G_{1} & =\frac{1}{2}\left[R_{2}+A(1-B)-A X\left(R_{1}-A B\right)\right], \quad F_{1}=\frac{1}{2} A,
\end{aligned}
$$




$$
\begin{aligned}
A_{2}= & A E G(A B X-A B E-B-1), \quad B_{2}=A F G[C-(1+C+A B F)] \\
H_{2}= & \frac{1}{2}[D G+A F G(A B X-A B E-B-1)+A C G(E-X) \\
& -A E G(1+C+A B F)], \quad G_{2}=\frac{1}{2} A E G\left(R_{1}-A B\right) \\
F_{2}= & \frac{1}{2}\left[A C(G-M-1)+A F G\left(R_{1}-A B\right)\right]
\end{aligned}
$$

The expressions appearing in (16) are

$$
\widehat{v}_{1}=(X-E) u_{*}-1, \quad \widehat{v}_{2}=\frac{R_{1}-A B+(A B X-A B E-B-1) u_{*}}{1+C+A B F}
$$

and

$$
\widehat{D}_{1 i}=\left|\begin{array}{ccc}
\widehat{A}_{i} & \widehat{H}_{i} & \widehat{G}_{i} \\
\widehat{H}_{i} & \widehat{B}_{i} & \widehat{F}_{i} \\
\widehat{G}_{i} & \widehat{F}_{i} & \widehat{C}_{i}
\end{array}\right|,
$$

with $\widehat{C}_{i}=0$ for $i=1,2$.

The Jacobian of (2) is

$$
J=\left(\begin{array}{cccc}
J_{11} & -(1+B) p & -p & -(1+C) p \\
A(1-B) u+A v & J_{22} & -A X u & A p-D u \\
q & (X-E) q & J_{33} & -F q \\
G v & \frac{G D}{A C} v+\frac{E G}{C} q & \frac{G F}{C} v+\frac{E G}{C} u & J_{44}
\end{array}\right)
$$

with

$$
\begin{aligned}
& J_{11}=R_{1}-A B p-B u-u-v-q-C v-H-A B p \\
& J_{22}=R_{2}-A B p-B u+A p-A X q-D v-B u-L, \\
& J_{33}=-1+p+X u-E u-F v-\Pi, \\
& J_{44}=-(1+M+N)+G p+G D(A C)^{-1} u+G F C^{-1} q .
\end{aligned}
$$

Stability is regulated by the eigenvalues of the Jacobian matrix (28), which for $Q^{(1)}$ are $R_{1}-H, R_{2}-L,-(1+\Pi),-(1+M+N)$, giving (17).

Those for $Q^{(2)}$, from which (18) follows, are

$$
\begin{array}{r}
H-R_{1}, \quad R_{2}-A B p^{(2)}+A p^{(2)}-L, \\
-1+p^{(2)}-\Pi, \quad-(1+M+N)+G p^{(2)} .
\end{array}
$$


For the disease-free equilibrium $Q^{(3)}$ the characteristic equation factors to give the two quadratic equations

$$
\begin{aligned}
x^{2}+A B p^{(3)} x+p^{(3)} q^{(3)} & =0, \\
x^{2}-\left(\hat{X}_{h}+\hat{Y}_{h}\right) x+\left(\hat{X}_{h} \hat{Y}_{h}-\frac{A E G}{C} p^{(3)} q^{(3)}\right) & =0,
\end{aligned}
$$

where

$$
\begin{aligned}
\hat{X}_{h} & =R_{2}+A-A B-A X q^{(3)}-L, \\
\hat{Y}_{h} & =-(1+M+N)+G p^{(3)}+G F C^{-1} q^{(3)} .
\end{aligned}
$$

Easily, the former (30) has always roots with negative real parts, since $A B p^{(3)}>0$ and $p^{(3)} q^{(3)}>0$. The Routh-Hurwitz condition for (31) give

$$
\hat{X}_{h}+\hat{Y}_{h}<0, \quad \hat{X}_{h} \hat{Y}_{h}-\frac{A E G}{C} p^{(3)} q^{(3)}>0 .
$$

To satisfy them we need to take $\hat{X}_{h}<0$ and $\hat{Y}_{h}<0$. In fact, if both $\hat{X}_{h}$ and $\hat{Y}_{h}$ are positive, the first condition would be violated, if instead $\hat{X}_{h}$ and $\hat{Y}_{h}$ have opposite signs the second condition would be violated. Thus (19) are found.

The characteristic equation for the predator-free equilibrium $Q^{(4)}$ again factors to give two explicit eigenvalues

$$
p^{(4)}+X u^{(4)}-E u^{(4)}-1-\Pi, \quad G p^{(4)}+G D(A C)^{-1} u^{(4)}-(1+M+N),
$$

and the quadratic,

$$
x^{2}+\bar{Q}_{h} x+\bar{R}_{h}=0
$$

where

$$
\begin{aligned}
\bar{Q}_{h} & =B u^{(4)}+A B p^{(4)}>0 \\
\bar{R}_{h} & =A B^{2} u^{(4)} p^{(4)}+\left(B p^{(4)}+p^{(4)}\right)\left(A u^{(4)}-A B u^{(4)}\right) .
\end{aligned}
$$

As before, the Routh-Hurwitz condition is $\bar{R}_{h}>0$, giving thus (20).

Also for $Q^{(5)}$ we have a factored form of the characteristic equation giving the quadratic

$$
x^{2}-\widetilde{C}_{h} x-\widetilde{F}_{h} \widetilde{Y}_{h}=0,
$$


where

$$
\tilde{Y}_{h}=\frac{G D}{A C} v^{(5)}, \quad \widetilde{C}_{h}=-B u^{(5)}, \quad \widetilde{F}_{h}=-D u^{(5)} .
$$

Hence we obtain explicit eigenvalues

$$
\begin{array}{r}
\widetilde{A}_{h}=R_{1}-(B+1) u^{(5)}-(1+C) v^{(5)}-H, \\
\widetilde{G}=-1+(X-E) u^{(5)}-F v^{(5)}-\Pi,
\end{array}
$$

and, since both $\widetilde{C}_{h}$ and $\widetilde{F}_{h} \widetilde{Y}_{h}$ are negative, (21) follow. 